\title{
The Estimation of Gastric Cancer Disease Status Using Array-Based Comparative Genomic Hybridization for Endoscopic Biopsy Specimens: Preliminary Experience (CGH for Gastric Cancer Biopsy)
}

Hideo Yanai, Tomoko Furuya, Takumi Furuya, Hiroto Hayashi, Kohsuke Sasaki

Department of Clinical Research; Department of Surgery,

National Hospital Organization Kanmon Medical Center, Shimonoseki, Japan

Department of Pathology, Yamaguchi University Graduate School of Medicine, Ube, Japan
Corresponding author:

Hideo Yanai, MD

Department of Clinical Research, National Hospital Organization Kanmon Medical Center, 1-1 Sotoura, Chofu, Shimonoseki, 752-8510, Japan

E-mail: yanaih@kanmon-mc2.hosp.go.jp

\section{ABSTRACT}

We used an aCGH mini-chip specific to gastric cancer for endoscopic biopsy specimens of gastric cancer to estimate the systemic disease status of the cancer before treatment. We employed the aCGH mini-chip to examine pretherapeutic endoscopic biopsy specimens of 32 gastric cancer lesions from 31 cases. The accuracy levels of the aCGH mini-chip for gastric cancer biopsy specimens were $44 \%$ for lymph node metastasis, $80 \%$ for liver metastasis, $76 \%$ for peritoneal dissemination, and $68 \%$ for invasion depth. The aCGH minichip was applicable for endoscopic gastric cancer biopsy specimens. It may contribute the therapeutic decision making with automatic evaluation of systemic gastric cancer disease status.

Abbreviation: aCGH, array-based comparative genomic hybridization

Keywords: Comparative genomic hybridization, Gastric cancer, Endoscopic biopsy

\section{BACKGROUND}

Since the 1960s, pathological evaluation of endoscopic biopsy specimens has been the standard for the diagnosis of gastric cancer. On the other hand, genomic instabilities such as anomalies of the DNA copy number have been explored as a new field in the study of cancer. From early analysis of aneuploidy using flow cytometry and chromosomal comparative genomic hybridization, array-based comparative genomic hybridization (aCGH) was developed. (1)

We have already reported an aCGH mini-chip for gastric cancer which was developed for the evaluation of gastric cancer disease status. (2) Using a DNA genome chip (MacArrayTM Karyo 1400, Macrogen, Korea) to examine 83 
surgically resected specimens of gastric cancer, we identified 138 clones closely related to the cancer disease status from a 1440 bacterial artificial chromsome (BAC) clone DNA library.

The aCGH mini-chip might enable the automatic and objective evaluation of the systemic cancer disease status. (fig. 1) In 30 patients with gastric cancer, we employed aCGH mini-chip to examine surgically resected gastric cancer specimens, and the accuracy rates were $66.7 \%$ for lymph node metastasis, $\mathbf{8 6 . 7 \%}$ for liver metastasis, $\mathbf{8 6 . 7 \%}$ for peritoneal dissemination, $96.7 \%$ for invasion depth (T1 or T2 over), and $72.4 \%$ for histologic type (intestinal or diffuse).

In the present study, we used an aCGH mini-chip specific to gastric cancer for endoscopic biopsy specimens of gastric cancer to estimate the systemic disease status of the cancer before treatment, and compared the aCGH results and clinicopathological results after resection.

\section{MATERIALS AND METHODS}

From October 2007 through January 2009, we employed the aCGH mini-chip to examine pretherapeutic endoscopic biopsy specimens of 32 gastric cancer lesions from 31 cases at the National Hospital Organization Kanmon Medical Center. This study was approved by the institutional review board. The ages of patients varied from 53 to 97 years (mean 70.5). They consisted of 20 males and 11 females, with 21 early (T1) lesions and 11 advanced (T2 or more) lesions.

In each case, we took one or two biopsy specimens through an endoscope in addition to specimens for histological diagnosis and stored them in a freezer at $-20^{\circ} \mathrm{C}$ until we examined the specimens using an aCGH mini-chip at the Department of Pathology of Yamaguchi University.

A tissue microdissection technique was used to reduce the contamination of samples by normal tissue components for array $\mathrm{CGH}$ analyses. Highmolecular-weight DNA was extracted from each microdissected tumor specimen with a DNA extraction kit (SepaGene, Sankojyunyaku Co., Tokyo, Japan) according to the manufacturer's instructions as previously described. (3) The development of the aCGH mini-chip dedicated for use with gastric cancer was previously reported. (2) (fig. 1) We evaluated the results obtained with the aCGH mini-chip for lymph node metastasis, liver metastasis, peritoneal

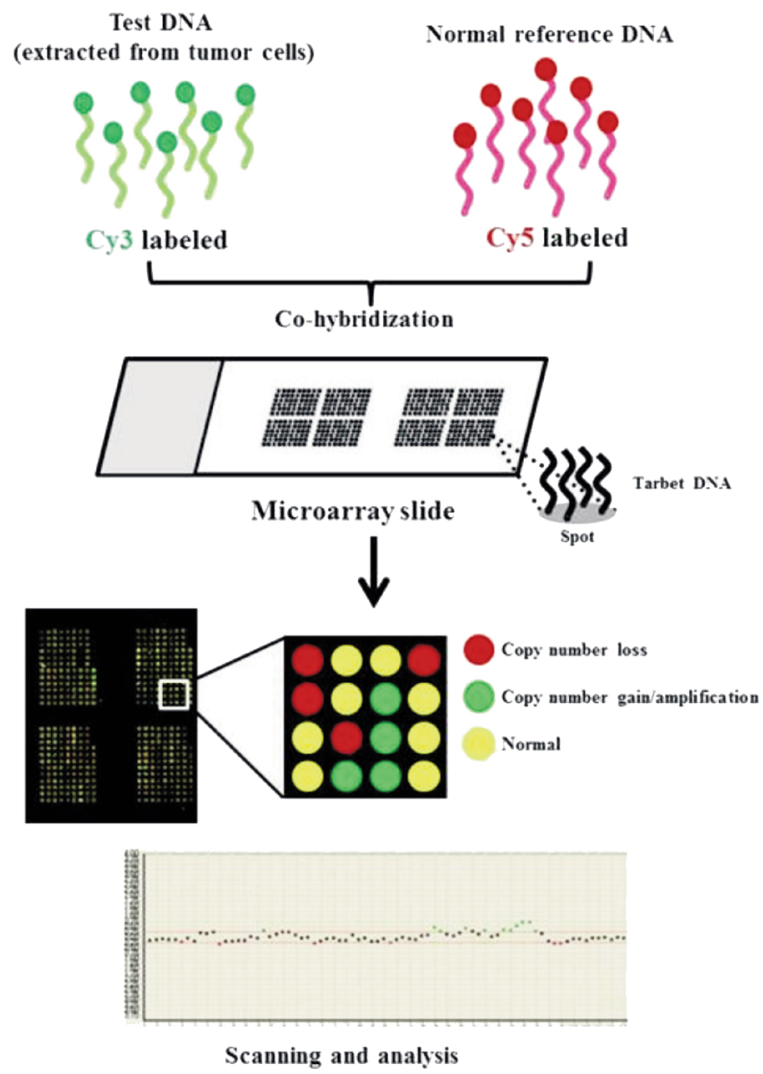

Figure 1 - Array CGH

Sample and gender matched reference genomic DNAs were labeled by the random priming method with fluorescence dyes, Cy3 and Cy5, respectively, and then were hybridized to the mini-chip aCGH slides. Images from hybridized slides are obtained by microarray scanner. The signal intensity ratios from individual spots are calculated, then displayed as a simple plot

dissemination, and invasion depth (T1 or T2 over) compared with clinicopathological findings after tumor resection.

\section{RESULTS}

aCGH analysis using the mini-chip for endosopic biopsy specimens was successfully completed for $78.1 \%$ (25 of 32 ) of the gastric cancer lesions. The incomplete results for $21.9 \%$ (7 of 32) of the specimens were due to defects in their freezing. Use of multiple specimens and a moist filter paper supporting the specimens solved this problem.

The accuracy levels of the aCGH mini-chip for gastric cancer biopsy specimens were $44 \%$ for lymph node metastasis, $80 \%$ for liver metastasis, $76 \%$ for peritoneal dissemination, and $68 \%$ for invasion depth (T1 or T2 over). (table 1) 


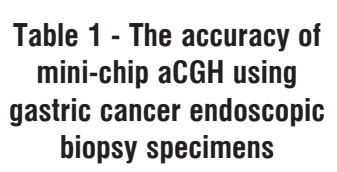

\section{DISCUSSION}

The results of the present study indicated that our mini-chip aCGH analysis was applicable to endoscopic gastric cancer biopsy specimens. As shown in the present study, it is possible to employ aCGH analysis using biopsy specimens to evaluate the whole disease status of gastric cancer with a very different approach from conventional microscopic examination for local pathological findings. aCGH analysis using a disease-specific miniarray is also expected to be useful for the automatic evaluation of cancer status. It may not only reduce the workload for pathologists but also contribute to therapeutic decision making by physicians and surgeons.

However, in this preliminary study, the accuracy rate was not as high as previous results for surgically resected specimens. (2) Intratumor heterogeneity did not affect the results, as shown in the previous study. However, endoscopic specimens are small and superficial, so intratumor heterogeneity may affect the aCGH results. Tissue microdissection technology is necessary in aCGH analysis because contamination of normal tissue reduces the analytical accuracy.

Recently, the main therapeutic modalities for gastric cancer consist of endoscopic resection, surgical operation and chemotherapy. (4) aCGH analysis may have some clinical impact for the choice of the therapeutic modality and measurement of the outcome in a different way from conventional pathological diagnosis. In the present study, we evaluated conventional clinicopathological factors such as lymph node metastasis, liver metastasis, peritoneal dissemination, and invasion depth. We hope that a mini-chip specific for lymph node metastasis will be useful to help determine whether to choose endoscopic submucosal dissection or natural orifice transluminal surgery for gastric cancer. (5)

\section{CONCLUSION}

The aCGH mini-chip was applicable for endoscopic gastric cancer biopsy specimens. It may reduce the workload of pathologists and contribute the therapeutic decision making of physicians and surgeons with automatic evaluation of systemic gastric cancer disease status.

\section{Author contributions}

Yanai H: study concept and design

Furuya To: aCGH

Furuya Ta: acquisition of data

Hayashi H: acquisition of data

Sasaki K: study supervision

\section{Conflict of interest}

The authors declare no competing interest.

\section{Funding}

This work was supported in part by grants from The Ministry of Education, Culture, Sports, Science, and Technology of Japan (20659055 and 21659088), The New Energy and Industrial Technology Development Organization (NEDO) of Japan, and Okinawa Prefecture. 
The Estimation of Gastric Cancer Disease Status Using Array-Based Comparative Genomic Hybridization for Endoscopic Biopsy Specimens

\section{REFERENCES}

1. Pinkel D, Segraves R, Sudar D, Clark S, Poole I, Kowbel D, et al. High resolution analysis of DNA copy number variation using comparative genomic hybridization to microarrays. Nat Genet. 1998 0ct;20(2):207-11.

2. Furuya T, Uchiyama T, Adachi A, Okada T, Nakao M, Oga A, et al. The development of a mini-array for estimating the disease state of gastric adenocarcinoma by array CGH. BMC Cancer. 2008 Dec 30;8:393.
3. Hashimoto Y, Oga A, Okami K, Imate Y, Yamashita Y, Sasaki K. Relationship between cytogenetic aberrations by $\mathrm{CGH}$ coupled with tissue microdissection and DNA ploidy by laser scanning cytometry in head and neck squamous cell carcinoma. Cytometry. 2000 Jun 1; 40(2):161-6.

4. Hartgrink HH, Jansen EP, van Grieken NC, van de Velde CJ. Gastric cancer. Lancet. 2009 Aug 8;374(9688):477-90.

5. Gotoda T. Endoscopic resection of early gastric cancer. Gastric Cancer. 2007;10(1):1-11. 